\title{
First principles study of structural phase stability of wide-gap semiconductors MgTe, MgS and MgSe
}

\author{
G. Gökoğlu ${ }^{\mathrm{a}, 1}$, M. Durandurdu ${ }^{\mathrm{b}}$, O. Gülseren ${ }^{\mathrm{a}, *}$ \\ a Department of Physics, Bilkent University, Ankara 06800, Turkey \\ ${ }^{\mathrm{b}}$ Department of Physics, University of Texas at El Paso, El Paso, TX 79968, USA
}

\section{A R T I C L E I N F O}

\section{Article history:}

Received 7 June 2009

Received in revised form 26 September 2009

Accepted 29 September 2009

Available online 27 October 2009

\section{PACS:}

61.50.Ah

71.15.Mb

81.05.Dz

61.50.Ks

Keywords:

Magnesium chalcogenides

Density functional theory

LDA and GGA functionals

Stability

\begin{abstract}
A B S T R A C T
The structural stability of different crystallographic phases of three magnesium chalcogenides, MgTe, $\mathrm{MgS}$ and MgSe, are investigated from first principles calculations based on density functional theory (DFT) using projector augmented waves (PAW) potentials within both generalized gradient approximation (GGA) and local density approximation (LDA). Five different phases of these compounds, rock-salt $(B 1)$, cesium chloride (B2), zinc-blende (B3), wurtzite $(B 4)$ and NiAs ( $\left.B 8_{1}\right)$, are considered in detail. LDA calculations of the ground state structure of all compounds considered are in accordance with previous studies, while calculations with GGA functionals resulted interesting perspectives. Ground state stable phases of MgS and MgSe are found as rock-salt structure, while the NiAs is the ground state of MgTe within the LDA scheme. But GGA calculations show that the ground states of MgTe and MgSe are wurtzite, while the ground state of MgS is same as in LDA calculations. There is also a structural transition observed for MgTe around $1.2 \mathrm{GPa}$ pressure from wurtzite to NiAs by GGA calculations. The calculated quantities show that the rock-salt-NiAs and zinc-blende-wurtzite pairs have similar energetics. The calculated structural quantities for all compounds under study in all phases agree well with the available experimental and theoretical values.
\end{abstract}

(c) 2009 Elsevier B.V. All rights reserved.

\section{Introduction}

The magnesium chalcogenides, MgSe, MgS and MgTe, are wideband gap semiconductors and have technological impact due to various applications in opto-electronics [1] and luminescent devices [2]. These compounds can crystallize in rock-salt (B1), zincblende (B3) and wurtzite (B4) structures. In addition to these phases, MgTe is believed to form the NiAs type (B8) structure. These materials have been studied extensively both experimentally and theoretically, but there are still unknowns and controversies about the their ground state structure and high pressure phases because of very small energetics difference between various structural phases.

Both MgSe and MgS are set forth in rock-salt structure as ground state under ambient pressure $[3,4]$. The stability of these compounds at high pressure is also investigated. From energy dispersive X-ray diffraction (EDXD) measurement under pressure, Ruoff et al. [3] showed that MgSe transforms from rock-salt (B1)

\footnotetext{
* Corresponding author. Tel.: +90 312 2901937; fax: +90 3122664579

E-mail address: gulseren@fen.bilkent.edu.tr (O. Gülseren).

1 Present address: Karabük University, Department of Physics, Karabük, 78050 Turkey.
}

to FeSi (B28) structure around $99 \mathrm{GPa}$ and approaches to sevenfold coordination at $202 \mathrm{GPa}$. Similarly, the rock-salt structure of $\mathrm{MgS}$ is found to be stable up to $54 \mathrm{GPa}[4]$ and no phase transformation has been reported in $\mathrm{MgS}$ to date. In addition, the zinc-blende structure is realized both for MgSe and MgS by epitaxial growth over GaAs substrate [5-7]. Besides, wurtzite structures of MgSe and $\mathrm{MgS}$ are also observed after annealing of their evaporated thin films by electron bombardment [8].

For the case of MgTe, experiments proposed two different structures. Early experimental studies showed that the ground state of MgTe is hexagonal wurtzite structure [9-13]. On the other hand, Li et al. [14] studied MgTe under pressure and proposed a phase transformation from wurtzite-to-a NiAs type structure at a pressure of $1-3.5 \mathrm{GPa}$. Upon releasing pressure, the NiAs type phase persists down to low pressures ( $\sim 0.2 \mathrm{GPa}$ ). Based on experimental and theoretical observations, the authors concluded that the thermodynamically stable phase of MgTe is indeed the NiAs type structure at ambient temperature and pressure. To date, the NiAs type MgTe has not been experimentally synthesized, to our knowledge.

These uncertainties and limited information about the physical properties, the ground state and high pressure phases of these compounds have stimulated several theoretical groups [15-32]. First of all, in the study where they discussed zinc-blende to wurtzite 
polytypism in detail, Yeh et al. [15] claimed that the ground state of MgTe is the NiAs structure based on first principles local density calculations. An early calculation on MgSe by the periodic Hartree-Fock method suggested that the wurtzite phase as the ground state at ambient conditions [16]. However, the $a b$ initio pseudopotential calculation [18] within local density approximation (LDA) is reported the rock-salt structure be more favorable over zinc-blende and wurtzite phases both for MgSe and MgS. The six different phases $(B 1, B 2, B 3, B 4, B 8$, and iron silicide (B28)) of MgSe and MgTe were studied by van Camp et al. in the framework of local density functional theory together with non-local norm-conserving pseudopotentials [19], and they found that rock-salt structure is stable for MgSe, while NiAs phase is the ground state of MgTe. Chaudhuri reported a study on high pressure properties of $\mathrm{B2}, \mathrm{B4}, \mathrm{B} 8$ and $\mathrm{PH}_{4} \mathrm{I}$ type structures of MgTe using LDA formalism [20]. They found the NiAs phase as ground state and a phase transition from $B 8$ to $\mathrm{PH}_{4} \mathrm{I}$ about $190 \mathrm{GPa}$ pressures. Moreover, MgTe, MgSe and MgS are studied by Chakrabarti [21] by plane-wave pseudopotential calculations and by Drief et al. [22] by full potential linearized augmented palne wave method (FP-LAPW) both within LDA. The electronic structures and elastic properties of MgS and MgSe are studied by Rached et al. [24] using full-potential linear muffin-tin orbitals (FP-LMTO) method while Rabah et al. [25] used FP-LAPW. Duman et al. presented a comprehensive study on electronic structure and dynamical properties of $B 1, B 3, B 4$ and $B 8$ phases of $\mathrm{MgS}$, $\mathrm{MgSe}$, and MgTe compounds [26]. These norm-conserving pseudopotential based LDA calculations also acclaimed NiAs structure for MgTe and rock-salt structure for MgSe and MgS as ground states phases. Recently, structural behaviours of magnesium chalcogenides under pressure are investigated on several studies. For example, Li et al. studied the NiAs and orthorhombic Pnma (B31) phases of MgTe [27]. They suggest the stability of NiAs at low pressure and a structural transition from $B 8$ to $B 31$ around $\sim 42$ GPa pressure from $a b$ initio phonon calculations. Moreover, pressure-induced structural transitions from $B 1$ to $B 28$ for $M g S e$ [28,29], from $B 1$ to $B 2$ for MgS [30] and again from B1 to B2 for MgTe, MgSe and MgS [31] are reported.

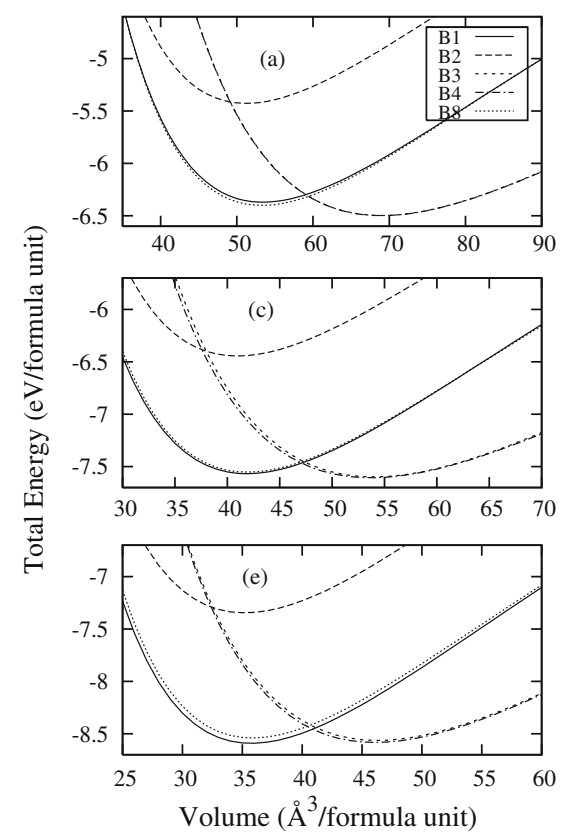

Most of these first principles calculations based on the LDA predict that the ground state phase for MgSe and MgS is the rock-salt structure and for MgTe is the NiAs type structure despite the variations in calculation scheme. Recently Cai et al. studied the NiAs and wurtzite phases of MgTe within both LDA and generalized gradient approximation (GGA) scheme [32]. They found the wurtzite and NiAs structures as ground states due to GGA and LDA formalisms, respectively. Their observed phase transition from wurtzite to NiAs is $\sim 1.1 \mathrm{GPa}$ which falls into the experimentally observed transition region. They also emphasized the underestimation of phase transition pressure in LDA calculations. Indeed their results question the validity of LDA approximation for the structural analysis of magnesium chalcogenides.

Since different crystallographic phases of related compounds have very similar energetics, a very detailed calculation is needed in order to distinguish the ground state structure and possible phase transitions. To our knowledge, there is not any comprehensive study on structural stability of several phases of magnesium chalcogenides using the generalized gradient approximation (GGA) together with projector augmented waves (PAW) potentials. The main goal of the present work is to clarify the stability of various crystal structures and possible phase transitions of these compounds between rock-salt, cesium chloride, zinc-blende, wurtzite and NiAs structures, in view of the calculated total energy and enthalpy curves at ground state (at $T=0$ ).

The rest of the paper is organized as follows: The details of the calculation method are explained in Section 2. The obtained results for each compound under study are presented and discussed at Section 3. Eventually paper concludes with a summary in Section 4.

\section{Computational methods}

The first principles total energy and electronic structure calculations presented have been performed using the plane-wave pseudopotential methods implemented in the Vienna $a b$ initio simulation package (VASP) [33] which is based on the density functional theory [34]. The ions are described by projector aug-

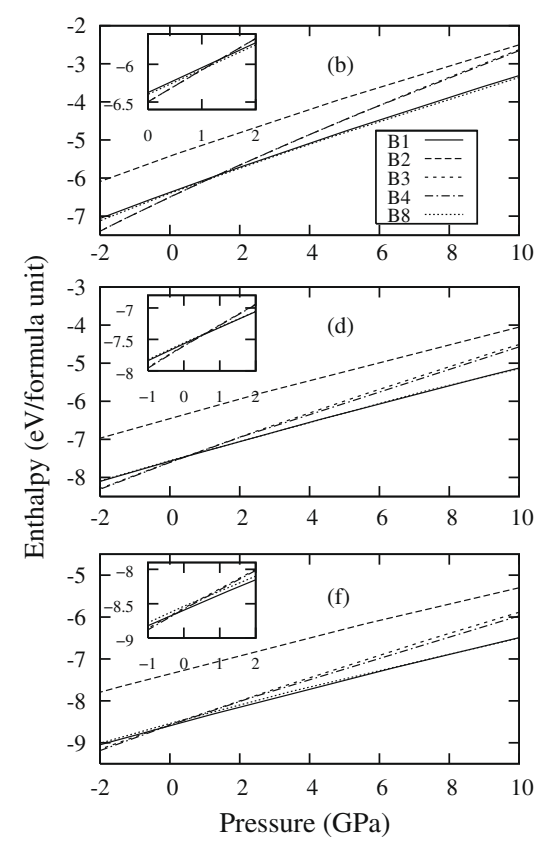

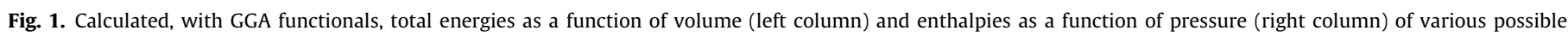

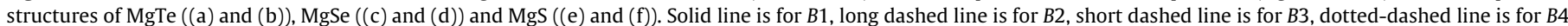

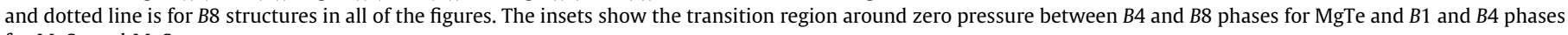
for MgSe and MgS. 

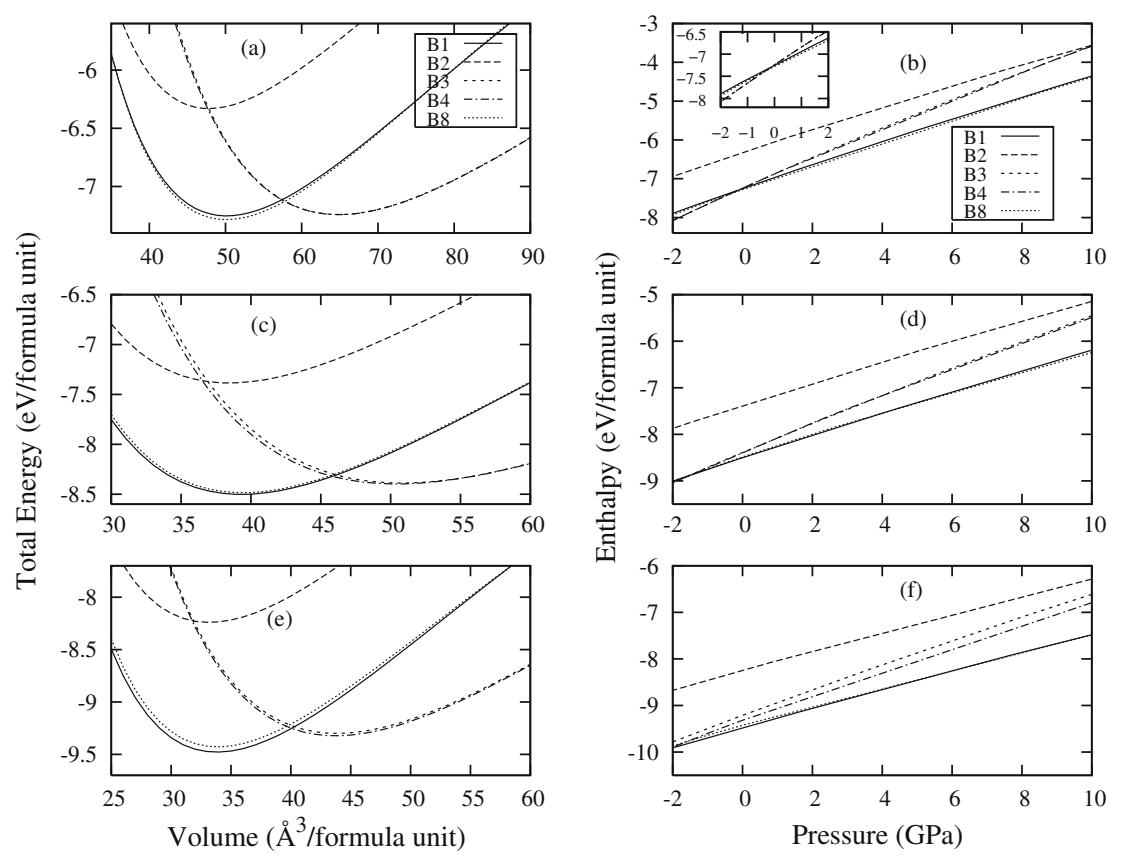

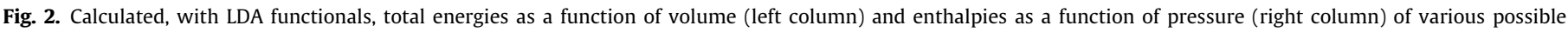

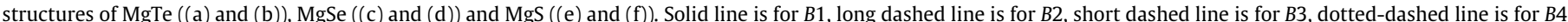

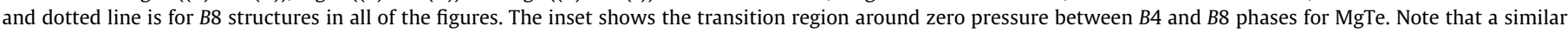
transition occurs at much negative pressures for MgSe and MgS.

mented waves (PAW) [35,36] potentials. Projector augmented wave approach is basically an all-electron method, which generalizes the pseudopotential method and the linear-augmented-planewave (LAPW) method and gives an advantage over usual planewave pseudopotential method by taking into account the core electrons. The electronic configurations of the atoms considered are as follows; Mg: $2 p^{6} 3 s^{2}$, Te: $5 s^{2} 5 p^{4}$, Se: $4 s^{2} 4 p^{4}$, S: $3 s^{2} 3 p^{4}$. Wave-functions were expanded in plane wave basis sets up to a kinetic energy cut-off value of $500 \mathrm{eV}$ which is determined to be sufficient in order to provide the energy convergence. The exchange correlation energy has been computed using both the local density approximation (LDA) [37] and the generalized gradient approximation (GGA) [38] functionals. Brillouin zone integrations is performed with special k-points within Monkhorst-Pack scheme [39] using tetrahedron method and Blöchl corrections [33]. We chose the k-points grids of $10 \times 10 \times 10$ for cubic structures (rock-salt, cesium chloride and zinc-blende) and $11 \times 11 \times 7$ for hexagonal structures (wurtzite and $\mathrm{NiAs}$ ). These $\mathbf{k}$-point grids give approximately same accuracy for all phases studied in this work. Internal crystal parameter $u$ is relaxed whenever it is allowed by symmetry (for example in wurtzite structures). Each point of the equation of states of hexagonal phases (wurtzite and NiAs structures) are constructed by minimizing the energy with respect to $c / a$ and $u$ at constant volume.

\section{Results and discussion}

In Figs. 1 and 2, calculated total energies as a function of volumes per formula unit and enthalpies as a function of pressure are shown for various possible structures of MgTe, MgSe and MgS by using GGA and LDA exchange-correlation functionals, respectively. The insets in the enthalpy figures show the transition region between the various phases around zero pressure. The general energetics of several phases show similar behavior for all compounds under study. B1 (rock-salt)-B8 (NiAs) and B3 (zincblende)-B4 (wurtzite) pairs have very similar equilibrium volumes as well as energy values. This zinc-blende to wurtzite polytypism [15] and similarity between rock-salt and NiAs structure [21] is discussed in detail in the literature before. This situation is directly

Table 1

The lattice parameters (in $\AA$ ), dimensionless $c / a$ ratio, bulk modulus (in GPa) and its dimensionless pressure derivative of several proposed structures of MgTe.

\begin{tabular}{|c|c|c|c|c|c|}
\hline & $a$ & $c$ & $c / a$ & $B_{0}$ & $B_{0}^{\prime}$ \\
\hline \multicolumn{6}{|c|}{ Rock-salt (B1) } \\
\hline GGA & 5.98 & & & 46.3 & 4.34 \\
\hline LDA & 5.85 & & & 53.4 & 4.23 \\
\hline Ref. [19] & 5.92 & & & 54.5 & 4.04 \\
\hline Ref. [22] & 5.86 & & & 52.0 & 4.10 \\
\hline Ref. [26] & 5.90 & & & 51.0 & 4.35 \\
\hline \multicolumn{6}{|c|}{ Cesium chloride (B2) } \\
\hline GGA & 3.71 & & & 45.9 & 4.28 \\
\hline LDA & 3.63 & & & 54.6 & 4.41 \\
\hline Ref. [19] & 3.68 & & & 49.5 & 4.20 \\
\hline \multicolumn{6}{|c|}{ Zinc-blende (B3) } \\
\hline GGA & 6.51 & & & 34.1 & 4.30 \\
\hline LDA & 6.38 & & & 39.0 & 4.19 \\
\hline Ref. [19] & 6.44 & & & 38.0 & 3.96 \\
\hline Ref. [22] & 6.38 & & & 38.7 & 3.89 \\
\hline Ref. [26] & 6.39 & & & 38.0 & 3.79 \\
\hline Exp. [13] & 6.36 & & & & \\
\hline \multicolumn{6}{|c|}{ Wurtzite (B4) } \\
\hline GGA & 4.61 & 7.48 & 1.620 & 34.0 & 4.32 \\
\hline LDA & 4.52 & 7.33 & 1.621 & 38.6 & 4.08 \\
\hline Ref. [19] & 4.53 & 7.41 & 1.635 & 42.8 & 3.82 \\
\hline Ref. [26] & 4.53 & 7.38 & 1.629 & 38.0 & 4.04 \\
\hline Exp. [10] & 4.53 & 7.38 & 1.629 & & \\
\hline Exp. [13] & 4.54 & 7.39 & 1.627 & & \\
\hline Exp. [14] & 4.55 & 7.39 & 1.625 & & \\
\hline \multicolumn{6}{|c|}{ Nickel arsenide (B8) } \\
\hline GGA & 4.23 & 6.86 & 1.620 & 47.5 & 4.37 \\
\hline LDA & 4.15 & 6.72 & 1.621 & 55.0 & 4.26 \\
\hline Ref. [19] & 4.18 & 6.84 & 1.635 & 58.3 & 3.89 \\
\hline Ref. [26] & 4.16 & 6.77 & 1.628 & 53.0 & 4.28 \\
\hline Exp. [14] & & & & $60.6 \pm 5.4$ & $4.1 \pm 0.3$ \\
\hline
\end{tabular}


Table 2

The lattice parameters (in $\AA$ ), dimensionless $c / a$ ratio, bulk modulus (in GPa) and its dimensionless pressure derivative of several proposed structures of MgSe.

\begin{tabular}{|c|c|c|c|c|c|}
\hline & $a$ & $c$ & $c / a$ & $B_{0}$ & $B_{0}^{\prime}$ \\
\hline \multicolumn{6}{|l|}{ Rock-salt (B1) } \\
\hline GGA & 5.51 & & & 61.0 & 4.14 \\
\hline LDA & 5.40 & & & 69.7 & 4.09 \\
\hline Ref. [3] & 5.50 & & & 68.1 & 4.08 \\
\hline Ref. [19] & 5.50 & & & 65.4 & 4.14 \\
\hline Ref. [22] & 5.40 & & & 68.3 & 4.15 \\
\hline Ref. [24] (GGA) & 5.52 & & & 68.4 & 3.79 \\
\hline Ref. [24] (LDA) & 5.40 & & & 74.8 & 3.52 \\
\hline Ref. [26] & 5.46 & & & 65.0 & 3.90 \\
\hline Exp. [3] & 5.47 & & & $62.8 \pm 1.6$ & $4.1 \pm 0.1$ \\
\hline \multicolumn{6}{|c|}{ Cesium chloride (B2) } \\
\hline GGA & 3.45 & & & 58.9 & 4.20 \\
\hline LDA & 3.37 & & & 67.9 & 4.12 \\
\hline Ref. [3] & 3.44 & & & 63.7 & 4.04 \\
\hline Ref. [19] & 3.44 & & & 62.5 & 4.10 \\
\hline \multicolumn{6}{|l|}{ Zinc-blende (B3) } \\
\hline GGA & 5.99 & & & 45.3 & 4.17 \\
\hline LDA & 5.88 & & & 51.1 & 4.04 \\
\hline Ref. [19] & 5.98 & & & 47.8 & 4.04 \\
\hline Ref. [22] & 5.87 & & & 50.5 & 4.02 \\
\hline Ref. [24] (GGA) & 6.02 & & & 48.1 & 4.17 \\
\hline Ref. [24] (LDA) & 5.89 & & & 54.8 & 4.27 \\
\hline Ref. [26] & 5.92 & & & 49.0 & 3.75 \\
\hline Exp. [5] & 5.89 & & & & \\
\hline \multicolumn{6}{|l|}{ Wurtzite (B4) } \\
\hline GGA & 4.28 & 6.76 & 1.578 & 43.9 & 4.32 \\
\hline LDA & 4.19 & 6.64 & 1.587 & 49.3 & 4.38 \\
\hline Ref. [18] & 4.14 & 6.81 & 1.643 & 48.2 & 4.10 \\
\hline Ref. [19] & 4.24 & 6.84 & 1.614 & 50.0 & 3.94 \\
\hline Ref. [24] (GGA) & 4.24 & 6.98 & 1.648 & 50.8 & 3.55 \\
\hline Ref. [24] (LDA) & 4.17 & 6.80 & 1.632 & 52.2 & 4.31 \\
\hline Ref. [26] & 4.20 & 6.83 & 1.627 & 50.0 & 3.78 \\
\hline Exp. [8] & 4.15 & 6.73 & 1.622 & & \\
\hline \multicolumn{6}{|c|}{ Nickel arsenide (B8) } \\
\hline GGA & 3.88 & 6.42 & 1.655 & 61.5 & 4.28 \\
\hline LDA & 3.80 & 6.31 & 1.661 & 70.6 & 4.20 \\
\hline Ref. [19] & 3.87 & 6.47 & 1.674 & 65.1 & 4.11 \\
\hline Ref. [26] & 3.82 & 6.37 & 1.667 & 67.0 & 4.15 \\
\hline
\end{tabular}

related to chemical bonding mechanism in these structures. Wurtzite and zinc-blende structures are fourfold coordinated, while rock-salt and NiAs structures are sixfold coordinated structures. The similarity in chemical bonding leads and the local structural arrangements up to the second neighbor distance lead similar energetics in these crystallographic phases. The energy of the $\mathrm{CsCl}$ (B2) structure is always higher than the others, indicating an unstable structure at ambient conditions, but there is a pressure induced phase transition to this structure at high pressures.

All calculated structural parameters of MgTe, MgSe and MgS together with available computational and experimental results are presented in Tables 1-3, respectively. The bulk modulus and its pressure derivatives are calculated using Vinet equation of states $[40,41]$. The pressure for each volume is calculated analytically from the first derivative of the Vinet equation according to volume. It should be noted that other computational data compiled in these tables are mostly LDA calculations. All quantities are in good agreement with the other works, but our calculated lattice constants from GGA calculations are slightly larger than the LDA results, as expected. The lower bulk modulus values obtained from GGA calculations are related to larger lattice constants. Note that, there is also a remarkable increase in lattice constants and a decrease in bulk modulus due to increase in atomic numbers of chalcogen atoms.

For MgTe, the minimum energies are realized with NiAs and wurtzite structures according to LDA and GGA functionals, respectively. But the minimum energy of the zinc-blende (B3) phase of MgTe is very close to the one of $B 4$ phase both with LDA and
Table 3

The lattice parameters (in $\AA$ ), dimensionless $c / a$ ratio, bulk modulus (in GPa) and its dimensionless pressure derivative of several proposed structures of $\mathrm{MgS}$.

\begin{tabular}{|c|c|c|c|c|c|}
\hline & $a$ & c & $c / a$ & $B_{0}$ & $B_{0}^{\prime}$ \\
\hline \multicolumn{6}{|l|}{ Rock-salt (B1) } \\
\hline GGA & 5.23 & & & 74.2 & 4.18 \\
\hline LDA & 5.13 & & & 84.0 & 4.04 \\
\hline Ref. [22] & 5.14 & & & 82.8 & 3.98 \\
\hline Ref. [24] (GGA) & 5.24 & & & 85.1 & 3.08 \\
\hline Ref. [24] (LDA) & 5.15 & & & 92.1 & 4.44 \\
\hline Ref. [26] & 5.18 & & & 81.0 & 4.15 \\
\hline Exp. [4] & & & & $78.9 \pm 3.7$ & $3.71 \pm 0.34$ \\
\hline \multicolumn{6}{|c|}{ Cesium chloride (B2) } \\
\hline GGA & 3.28 & & & 69.2 & 3.82 \\
\hline LDA & 3.21 & & & 77.6 & 3.76 \\
\hline \multicolumn{6}{|l|}{ Zinc-blende (B3) } \\
\hline GGA & 5.70 & & & 55.9 & 4.11 \\
\hline LDA & 5.60 & & & 62.0 & 4.17 \\
\hline Ref. [22] & 5.61 & & & 61.2 & 4.06 \\
\hline Ref. [24] (GGA) & 5.72 & & & 60.0 & 3.89 \\
\hline Ref. [24] (LDA) & 5.62 & & & 66.5 & 4.49 \\
\hline Ref. [26] & 5.64 & & & 60.0 & 4.06 \\
\hline Exp. [5] & 5.62 & & & & \\
\hline Exp. [7] & 5.66 & & & & \\
\hline \multicolumn{6}{|l|}{ Wurtzite (B4) } \\
\hline GGA & 4.07 & 6.42 & 1.577 & 56.1 & 4.27 \\
\hline LDA & 4.00 & 6.31 & 1.577 & 62.7 & 4.17 \\
\hline Ref. [18] & 3.95 & 6.44 & 1.633 & 57.0 & 4.10 \\
\hline Ref. [24] (GGA) & 4.05 & 6.59 & 1.628 & 59.6 & 3.92 \\
\hline Ref. [24] (LDA) & 3.97 & 6.49 & 1.634 & 64.9 & 2.96 \\
\hline Ref. [26] & 4.00 & 6.49 & 1.625 & 63.0 & 4.18 \\
\hline Exp. [8] & 3.97 & 6.31 & 1.588 & & \\
\hline Exp. [42] & 3.97 & 6.44 & 1.622 & & \\
\hline \multicolumn{6}{|c|}{ Nickel arsenide (B8) } \\
\hline GGA & 3.67 & 6.15 & 1.679 & 74.6 & 4.29 \\
\hline LDA & 3.60 & 6.05 & 1.680 & 84.7 & 4.21 \\
\hline Ref. [21] & 3.60 & 6.00 & 1.666 & & \\
\hline Ref. [26] & 3.61 & 6.09 & 1.690 & 82.0 & 4.15 \\
\hline
\end{tabular}

GGA calculations and two curves almost overlap for all volume range. The prediction of NiAs ground state of MgTe within LDA is in agreement with previous theoretical results $[19,20,26]$ whose calculations are also based on local density formalism. On the other hand, GGA calculations of MgTe suggest the wurtzite structure as ground state, which is also in agreement with recently published GGA calculation of Cai et al. [32] and earlier experiments. The experimental studies are very limited because of the high hygroscopic properties of this compound [9,10,14].

The structural stability of several phases can be better established with calculated Gibbs free energies. Gibbs free energy is defined as: $G=E+p V-T S$. Our density functional calculations are essentially performed at zero temperature and entropic contributions can be neglected. Therefore, the enthalpy values, $H=E+p V$, including the pressure-volume effects are calculated. According to LDA calculations (Fig. 2), NiAs phase of MgTe has the lowest enthalpy at zero pressure, but wurtzite structure can be stabilized at slightly negative pressures $(\sim-0.4 \mathrm{GPa})$, which correspond to the expanded lattices with higher lattice constants than the equilibrium state. NiAs structure of MgTe is always stable at elevated pressures according to LDA data.

The enthalpy curves from GGA calculations of MgTe show somewhat different behavior. Clearly, at zero pressure, wurtzite structure has lower enthalpy than the all other phases and a pressureinduced structural transition from wurtzite to NiAs occurs around $\sim 1.1 \mathrm{GPa}$ pressure as seen from the inset and NiAs structure becomes more stable at elevated pressures. This predictions including phase transition pressure are in excellent agreement with the experimental results of Li et al. [14] who suggest a transition pressure $1-3.5 \mathrm{GPa}(\mathrm{WZ} \Rightarrow \mathrm{NiAs})$ and GGA calculation of Cai et al. [32]. 
Table 4

Total energies (meV/formula unit) with respect to the ground state energy for various possible structures of MgTe, MgSe, and MgS.

\begin{tabular}{|c|c|c|c|c|c|c|}
\hline & \multicolumn{2}{|l|}{ MgTe } & \multicolumn{2}{|l|}{ MgSe } & \multicolumn{2}{|l|}{ MgS } \\
\hline & GGA & LDA & GGA & LDA & GGA & LDA \\
\hline Rock-salt & 128 & 31 & 37 & 0 & 0 & 0 \\
\hline Cesium chloride & 1072 & 953 & 1161 & 1117 & 1245 & 1238 \\
\hline Zinc-blende & 1 & 43 & 7 & 114 & 28 & 178 \\
\hline Wurtzite & 0 & 42 & 0 & 105 & 6 & 154 \\
\hline Nickel arsenide & 96 & 0 & 53 & 17 & 10 & 49 \\
\hline
\end{tabular}

For the case of MgSe, the GGA and LDA calculations also suggest a different structure as ground state, as observed in MgTe. According to LDA calculations (Fig. 2), ground state phase seems to be rock-salt (B1) structure. On the other hand, wurtzite is the ground state of MgSe due to GGA with slightly lower enthalpy than B1 phase at zero pressure (Fig 1). The GGA data also concerns a structural phase transition from wurtzite to rock-salt at $\sim 0.5 \mathrm{GPa}$ and rock-salt phase is stabilized at higher pressures. Enthalpy values of rock-salt and NiAs phases of MgSe almost overlap in all pressure range. In previous studies, the ground state of $\mathrm{MgSe}$ is predicted to be rock-salt [19] and wurtzite [16] by pseudopotential LDA and
Hartree-Fock calculations, respectively. There is only a few study on MgSe compound based on GGA exchange-correlation functionals, which seem to be more successful to describe the ground state and its physical properties in several binary compounds than LDA scheme.

Ground state stable phase of MgS compound is found to be rock-salt without any discrepancy using both LDA and GGA functionals which is also in agreement with the previous theoretical results [26]. The obtained ground state structures and relative energetics of various structures of all compounds under study due to LDA and GGA calculations are summarized in Table 4.

As emphasized by Cai et al. [32], a key point about the stability of these compounds are the ionicity of chemical bonding. The ground state stable phases of several compounds can be foreseen by taking into account the ionicity of chemical bonding in related crystal structures. In this view, MgTe compound can only be stable in fourfold coordinated wurtzite structure with low spectroscopic ionicity parameter (0.554), while MgSe and MgS can form rock-salt and wurtzite structures. For further evidence, our GGA calculations are also repeated using ultrasoft pseudopotentials rather than PAW potentials and the same results are obtained for ground state stable phases. The LDA calculations in the literature indicate that the LDA functionals are not adequate in describing the physical properties
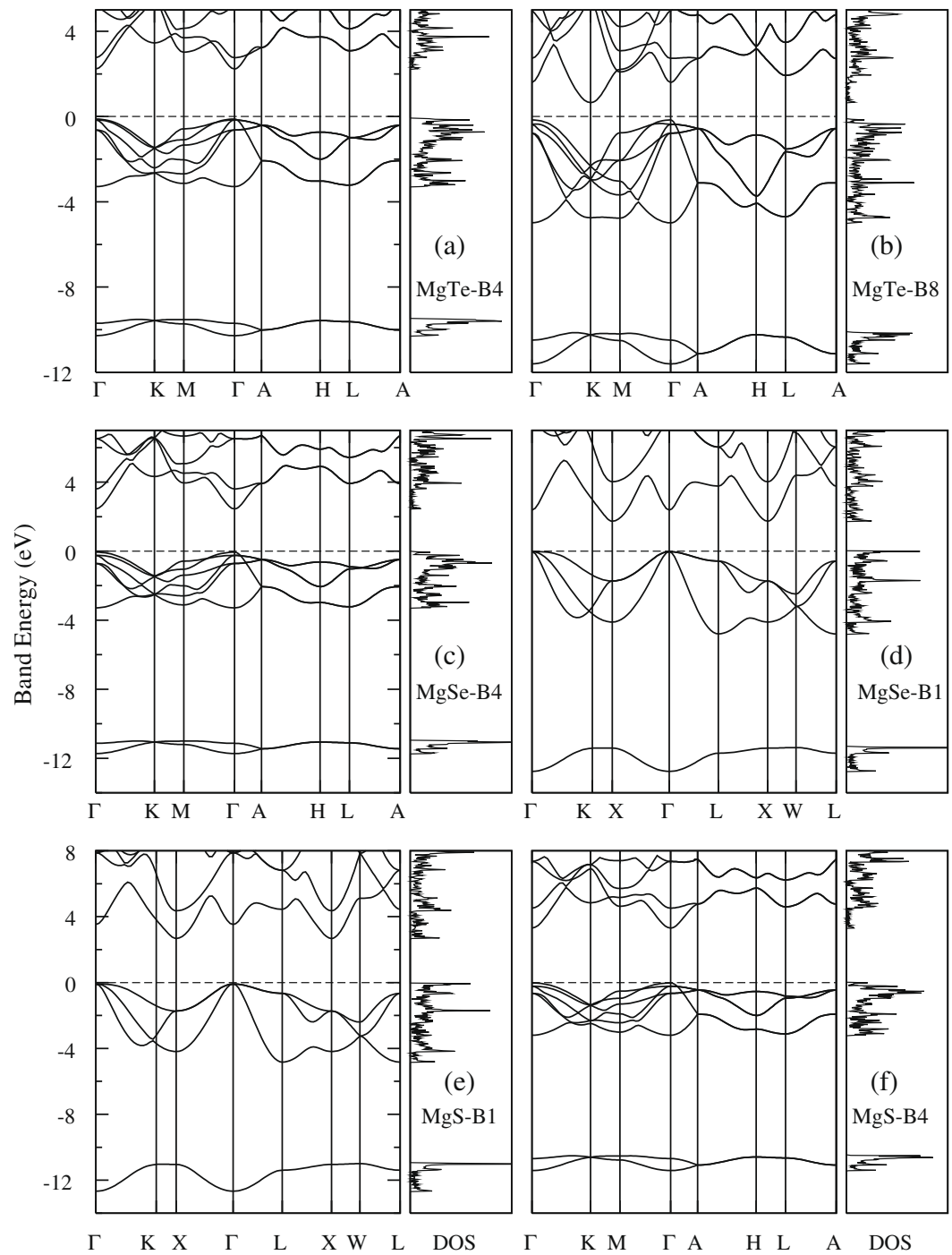

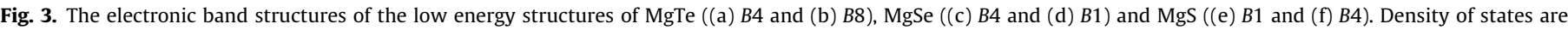
included on the right of band structure figures. 
of these compounds, since possible phase transitions occurring at low pressures between fourfold and sixfold coordinated structures cannot be distinguished. On the other hand, results of calculations with GGA functionals are more consistent with the experiments.

The electronic band structures of the two low energy structures of the compounds under study (MgTe, MgSe and MgS) calculated with GGA functional are shown in Fig 3. In total energy-volume curves, there are two minima with slightly different volumes. The lower energy phase of each region is identified and the corresponding band structure is presented in Fig. 3. The general characteristics of energy bands are similar for the same structure of three compounds, but the energy of low energy $s-p$ bands of MgTe lie $\sim 2 \mathrm{eV}$ above the same bands of other two compounds. The lowest band gap value is found in $B 1$ phase for all compounds, this is an indirect gap along $\Gamma-X$. Specially for MgTe compound, this indirect gap of $B 1$ phase become extremely low $(0.44 \mathrm{eV})$ in comparison to other compounds where it is approximately $2 \mathrm{eV}$ larger.

The energy gaps in $B 1$ and $B 8$ phases are indirect, while there are direct band gaps in $B 3$ and $B 4$ phases at $\Gamma$-point. This situation is also related to arrangement of chemical bonding in these phases since $B 1$ and $B 8$ are sixfold, $B 3$ and $B 4$ are fourfold coordinated structures. These findings are in great agreement with the LDA calculated bands of Duman et al. [26]. However, the only exception is that, in our GGA results, the $B 8$ phase of MgSe compound shows a direct band gap in which the band energy at $\Gamma$-point is slightly lower than the that of k-point. Our GGA calculations of band gaps of zinc-blende phases are 2.32, 2.56 and $3.36 \mathrm{eV}$ for MgTe, MgSe and $\mathrm{MgS}$, respectively, these values are $1.15 \mathrm{eV}$ smaller than the experimentally measured values. LDA results of Ref. [26] also needs approximately same amount of opening of band gap in order to compare to the experiments.

\section{Conclusions}

We presented and discussed the structural stability of $B 1, B 2$, $B 3, B 4$, and $B 8$ phases of $\mathrm{MgTe}, \mathrm{MgSe}$, and $\mathrm{MgS}$ wide-gap semiconductor compounds in the framework of density functional theory using both LDA and GGA functionals with projector augmented waves (PAW) potentials. The obtained stable phases of these compounds are summarized in Table 4. Determining the ground state structure of magnesium chalcogenides is not obvious because of tiny energy differences between the different structures, hence very detailed and accurate total energy calculations are necessary in order to determine the stable phase and possible phase transition pressures. Our GGA calculations show the wurtzite structure as stable phases of MgTe and MgSe compounds. The obtained phase transition pressure $(\sim 1.1 \mathrm{GPa})$ of MgTe from wurtzite to $\mathrm{NiAs}$ phase is in excellent agreement with experimental data (13.5 GPa). The stable phase of $\mathrm{MgS}$ is $B 1$ structure due to both LDA and GGA formalisms. Our GGA calculated electronic band structures are also in very good agreement with available theoretical calculations.

\section{Acknowledgments}

This research was supported in part by TÜBITAK (The Scientific and Technological Research Council of Turkey) through TR-Grid e-
Infrastructure Project, part of the calculations has been carried out at ULAKBIM Computer Center. G.G. acknowledges TÜBITAK for research fellowship under program-2218 and OG acknowledges the support of Turkish Academy of Sciences, TÜBA.

\section{References}

[1] M.W. Wang, M.C. Phillips, J.F. Swenberg, E.T. Yu, J.O. McCaldin, T.C. McGill, J. Appl. Phys. 73 (1993) 4660.

[2] R. Pandey, S. Sivaraman, J. Phys. Chem. Solids 52 (1991) 211.

[3] A.L. Ruoff, T. Li, A.C. Ho, M.F. Pai, H. Luo, R.G. Greene, C. Narayana, J.C. Molstad, S.S. Trail, F.J. DiSalvo, P.E. van Camp, Phys. Rev. Lett. 81 (1998) 2723.

[4] S.M. Peiris, A.J. Campbell, D.L. Heinz, J. Phys. Chem. Solids 55 (1994) 413.

[5] H. Okuyama, K. Nakano, T. Miyajima, K. Akimato, J. Cryst. Growth 117 (1992) 139.

[6] H. Okuyama, K. Nakano, T. Miyajima, K. Akimato, Jpn. J. Appl. Phys. 30 (1991) L1620.

[7] L. Konczenwicz, P. Bigenwald, T. Cloitre, M. Chibane, R. Ricou, P. Testud, O. Briot, R.L. Aulombard, J. Cryst. Growth 159 (1996) 117.

[8] H. Mittendorf, Z. Phys. 183 (1965) 113.

[9] W. Zachariasen, Z. Phys. Chem. 128 (1927) 417.

[10] W. Klemm, K. Wahl, Z. Anorg. Allg. Chem. 266 (1951) 289.

[11] A. Kuhn, A. Chevy, M.-J. Naud, J. Cryst. Growth 9 (1971) 263.

[12] S.G. Parker, A.R. Reinberg, J.E. Pinnell, W.C. Holton, J. Electrochem. Soc. 118 (1971) 979.

[13] A. Waag, H. Heinke, S. Scholl, C.R. Becker, G. Landwehr, J. Cryst. Growth 131 (1993) 607.

[14] T. Li, H. Luo, R.G. Greene, A.L. Ruoff, S.S. Trail, F.J. Disalvo, Phys. Rev. Lett. 74 (1995) 5232.

[15] C. Yeh, Z.W. Lu, S. Froyen, A. Zunger, Phys. Rev. B 46 (1992) 10086.

[16] R. Pandey, A. Sutjianto, Solid State Commun. 91 (1994) 269.

[17] G. Kalpana, B. Palanivel, R.M. Thomas, M. Rajagopalan, Physica B 222 (1996) 223.

[18] S.G. Lee, K.J. Chang, Phys. Rev. B 52 (1995) 1918.

[19] P.E. Van Camp, V.E. Van Doren, J.L. Martins, Phys. Rev. B 55 (1997) 775

[20] C.B. Chaudhuri, G. Pari, A. Mookerjee, A.K. Bhattacharyya, Phys. Rev. B 60 (1999) 11846.

[21] A. Chakrabarti, Phys. Rev. B 62 (2000) 1806.

[22] F. Drief, A. Tadjer, D. Mesri, H. Aourag, Catal. Today 89 (2004) 343.

[23] F. Marinelli, H. Dupin, A. Lichanot, J. Phys. Chem. Solids 61 (2000) 1707.

[24] D. Rached, N. Benkhettou, B. Soudini, B. Abbar, N. Sekkal, M. Driz, Phys. Stat. Sol. (b) 240 (2003) 565.

[25] M. Rabah, B. Abbar, Y. Al-Douri, B. Bouhafs, B. Sahraoui, Mater. Sci. Eng. B 100 (2003) 163.

[26] S. Duman, S. Bağcı, H.M. Tütüncü, G.P. Srivastava, Phys. Rev. B 73 (2006) 205201.

[27] Y. Li, Y. Ma, T. Cui, Y. Yan, G. Zou, Appl. Phys. Lett. 92 (2008) 101907.

[28] F.A. Sahraoui, F. Arab, S. Zerroug, L. Louail, Comput. Mater. Sci. 41 (2008) 538

[29] M. Ozduran, M. Durandurdu, EPL 84 (2008) 56001.

[30] Y.-D. Guo, Z.-J. Yang, Q.-H. Gao, W. Dai, Physica B 403 (2008) 2367.

[31] D. Varshney, N. Kaurav, U. Sharma, R.K. Singh, J. Phys. Chem. Solids 69 (2008) 60.

[32] Y. Cai, S. Wu, J. Yu, R. Xu, Phys. Rev. B 74 (2006) 214112.

[33] Computations have been carried out with the VASP software G. Kresse, J. Hafner, Phys Rev. B 47 (1993) R558;

G. Kresse, J. Furthmüller, Phys Rev. B 54 (1996) 11169.

[34] W. Kohn, L.J. Sham, Phys. Rev. 140 (1965) A1133; P. Hohenberg, W. Kohn, Phys. Rev. 136 (1964) B864.

[35] P.E. Blöchl, Phys. Rev. B 50 (1994) 17953.

[36] G. Kresse, D. Joubert, Phys. Rev. B 59 (1999) 1758.

[37] J.P. Perdew, A. Zunger, Phys. Rev. B 23 (1981) 5048.

[38] J.P. Perdew, Y. Wang, Phys. Rev. B 45 (1992) 13244; J.P. Perdew, Y. Wang, Phys. Rev. B 46 (1992) 6671.

[39] H.J. Monkhorst, J.D. Pack, Phys. Rev. B 13 (1976) 5188.

[40] P. Vinet, J. Ferrante, J.R. Smith, J.H. Rose, J. Phys. C: Solid State Phys. 19 (1986) L467;

P. Vinet, J.H. Rose, J. Ferrante, J.R. Smith, J. Phys.: Condens. Matter. 1 (1987) 1941.

[41] R.E. Cohen, O. Gülseren, R.J. Hemley, Am. Mineral. 85 (2000) 338.

[42] P. Villars, L.D. Calvert, Pearson's Handbook of Crystallographic Data for Intermetallic Phases, American Society of Metals, Metals Park, OH, 1985. 